\title{
BRAIN NEOPLASMS
}

\section{ETOPOSIDE THERAPY FOR RECURRENT GLIOMAS}

The efficacy and toxicity of long-term salvage chemotherapy with oral etoposide was studied in 14 children with recurrent supratentorial gliomas at the Department of Neurosciences, University of California, San Diego. All children had received radiotherapy and nitrosourea chemotherapies previously. MRI showed either a partial response or stable disease over a median duration of 8 months in 7 (50\%). Toxic effects included alopecia ( 8 children), diarrhea (6), weight loss (4), anemia (4), neutropenia (4), and thrombocytopenia (4). (Chamberlain MC. Recurrent supratentorial malignant gliomas in children. Long-term salvage therapy with oral etoposide. Arch Neurol May 1997;54:554-558). Respond: Marc C Chamberlain MD, University of California, San Diego, 9500 Gilman Dr, 0812, La Jolla, CA 92093).

COMMENT. Etoposide appears to be of some value in the salvage therapy of recurrent malignant supratentorial gliomas, and its toxicity is relatively low. This agent is also of value in the treatment of recurrent cerebellar gliomas. (Chamberlain MC. I Child Neurol 1997;12:200-204).

\section{CEREBELLAR MUTISM AFTER POSTERIOR FOSSA SURGERY}

Six children, aged 6 to 12 years, who developed cerebellar mutism after surgery for a posterior fossa mass or as a result of trauma are reported from the Children's Hospital, Los Angeles, CA. The incidence following posterior fossa tumor resection was $1.6 \%$. Four had primitive neuroectodermal tumors (medulloblastoma), a fifth had mutism following trauma, and the sixth had an arteriovenous malformation. Hydrocephalus developed as a result of the tumor or AVM in 4 patients. Cerebellar mutism duration was 10 days in one patient and 2 to 8 weeks in the others. Dysarthria occurred during recovery in 4 . Trauma to the dentate nucleus and/or the superior cerebellar peduncle is suggested as the cause of reversible mutism. (Koh S, Turkel SB, Baram TZ. Cerebellar mutism in children: report of six cases and potential mechanisms. Pediatr Neurol April 1997;16:218-219). (Respond: Dr Koh, Children's Hospital Los Angeles, Mailstop \#82, 4650 Sunset Blvd, Los Angeles, CA 90027).

COMMENT. Cerebellar mutism is reviewed in Progress in Pediatric Neurology III, 1997;pp306-307). This complication of posterior fossa tumor resection is correlated with the amount of posterior vermis resected, adherence of the tumor to the floor of the fourth ventricle, shunting for hydrocephalus prior to surgery, and postsurgical edema of the pontine tegmentum. More than 50 cases are cited in the literature.

\section{VASCULAR DISORDERS: CAUSES OF STROKE}

Thirty five cases of stroke are reported from the CHU Timone, Marseille, France. Specific causes included sickle cell disease, cardiopathy, migraine, HIV infection, Moyamoya syndrome, Williams syndrome, homocystinuria, lupus anticoagulant, protein C deficiency, MELAS, and pheochromocytoma. Associated conditions were head injury, surgery, angiography, oral contraceptive, infection, and hypertension. Hemichorea was the presenting sign in 3. (Mancini J, Girard N, Chabrol B et al. Ischemic cerebrovascular disease in children: retrospective study of 35 patients. I Child Neurol April 1997;12:193-199). (Respond: Dr Mancini, CHU Timone, 13385 Marseille, Cedex 5, France). 